\title{
A Control Scheme for Stable Force-Reflecting Teleoperation over IP Networks
}

\author{
Ilia G. Polushin, Peter X. Liu, and Chung-Horng Lung \\ Department of Systems and Computer Engineering \\ Carleton University \\ Ottawa, ON, K1S 5B6, Canada \\ \{polushin, xpliu, chlung\}@sce.carleton.ca
}

\begin{abstract}
The problem of force-reflecting teleoperation over IP networks is addressed. The existence of time-varying communication delay and possibility of data packets dropouts are taken into consideration. Since significant data dropouts may result in discontinuity of the reference trajectory transmitted through the communication channel, the proposed control scheme includes a filter that provides a smooth approximation of a possibly discontinuous reference trajectory. The stability of the overall system is guaranteed by a version of the IOS small gain theorem for functional-differential equations. It is also shown that, in the case of reliable communication protocols, the proposed scheme guarantees that the slave manipulator tracks the delayed trajectory of the master with a prescribed small error.
\end{abstract}

Index Terms - Force-Reflecting Teleoperation, Time Delay, Stabilization

\section{Introduction}

Bilateral (or force-reflecting) teleoperation is a challenging area of modern technology with a number of traditional and potential applications, including space and undersea exploration, handling of hazardous materials, high-precision assembly, and robotic telesurgery. Recently, essential research interest has been attracted by using the Internet as a communication medium for teleoperation. When teleoperation is performed over the Internet, the signals transmitted between the master and the slave manipulators are subject to communication delay. In some exceptional cases (for example, when a network with guaranteed quality of service (QoS) is utilized, where there are no network congestions or node failures), the communication delay may be known and constant or vary very little, however, in the general situation the communication delay is time-varying and unknown. It is a well-known fact that the existence of a time delay in the communication channel may cause the force-reflecting teleoperator system to be unstable [1]. To the date, several control schemes are proposed in the literature that overcome the delay-induced instability (see [2], [3], [4], and the survey [5], among others) however, only little work address the problem under the assumption that the delay is time-varying [6], [7]. Another important feature of the Internet-based teleoperation is a possibility of data packet dropouts. When a congestion occurs in the network, and some packets are lost, it may be advantageous to forget the old packet and transmit a new one which contains the recent information (see for example [8]). If a significant amount of data is dropped, it may result in discontinuity of the reference trajectories and the forces transmitted between the master and the slave. To the best of our knowledge, this problem was not addressed in the literature in the context of stability and tracking performance of force-reflecting teleoperator systems.

In this paper, we propose a control scheme that guarantees stable force-reflecting teleoperation in the presence of time-varying communication delay and data packets dropouts. The important feature of the scheme proposed is that it incorporates a "dirty-derivative" filter on the slave side. The purpose of introducing the filter is twofold. First, when there are no packets dropouts, the reference trajectory is smooth, and the filter works as an observer giving estimates for the velocity and accelerations of the master trajectory. On the other hand, when the reference signal is discontinuous (this may happen if the data packets dropout is essential), the filter operates as a "governor" providing a smooth approximation of the discontinuous reference trajectory. The stability of the overall scheme is investigated using the IOS small gain theorem for functional differential equations previously proposed in [9]. For the case of time-varying but smooth enough communication delay we show that the proposed control scheme guarantees the stability of the overall system and, additionally, that the slave tracks the delayed master trajectory with an arbitrarily small tracking error. When communication delay is a discontinuous function (this may happen if a significant amount of data is dropped), it may result in a discontinuous reference trajectory for the slave manipulator, which destroys the tracking properties of the system, however, a form of the input-to-output stability of the overall scheme can still be guaranteed.

The paper is organized as follows. The mathematical model of the teleoperator system is described in section II. The control scheme is introduced in section III. In section IV, our main results are presented. Some computer simulations of the proposed control scheme are presented in section $\mathrm{V}$, while the conclusions are given in section VI. Finally, Appendix A contains a necessary 
definition. Proofs are omitted due to space reasons.

\section{Mathematical Model of the Telerobotic System}

\section{A. Master and Slave Manipulators}

Let us consider a teleoperator system where both the master and the slave are $n$-DOF manipulators described by Euler-Lagrange equations of the form

$$
\begin{gathered}
H_{m}\left(q_{m}\right) \ddot{q}_{m}+C_{m}\left(q_{m}, \dot{q}_{m}\right) \dot{q}_{m}+G_{m}\left(q_{m}\right) \\
=u_{m}+f_{h}+\hat{f}_{e} \\
H_{s}\left(q_{s}\right) \ddot{q}_{s}+C_{s}\left(q_{s}, \dot{q}_{s}\right) \dot{q}_{s}+G_{s}\left(q_{s}\right)=u_{s}+f_{e}
\end{gathered}
$$

where $q_{i}, \dot{q}_{i}$ are positions and velocities of the master $(i=m)$ and the slave $(i=s)$, manipulators respectively. We assume that $q_{m} \in \mathbb{Q}_{m}, q_{s} \in \mathbb{Q}_{s}$, where both configuration spaces $\mathbb{Q}_{m}, \mathbb{Q}_{s}$ are assumed to be compact subsets of $\mathbb{R}^{n}$. Further, $H_{m}\left(q_{m}\right), H_{s}\left(q_{s}\right)$ are inertia matrices, $C_{m}\left(q_{m}, \dot{q}_{m}\right), C_{s}\left(q_{s}, \dot{q}_{s}\right)$ are matrices of centrifugal and Coriolis forces, and $G_{m}\left(q_{m}\right), G_{s}\left(q_{s}\right)$ are vectors of potential forces of the master and the slave manipulators respectively. Also, $f_{h} \in \mathbb{R}^{n}$ is a force applied by the human operator to move the master manipulator, $f_{e} \in \mathbb{R}^{n}$ is the contact force due to the environment applied to the slave, and $\hat{f}_{e} \in \mathbb{R}^{n}$ is the force applied to the motors of the master that reflects the contact force due to environment on the slave side. Finally, $u_{m}, u_{s} \in \mathbb{R}^{n}$ are the control inputs of the master and the slave respectively. It is assumed throughout the paper that the dynamics of the master and the slave manipulators (1), (2) possess several standard properties (see, for example [10, Section 2.1]).

\section{B. Environment}

In this paper, we assume that the environment in contact with the slave can be described as an unknown dynamical system satisfying the so called input-to-output stability condition [11]. More precisely, our assumption is as follows.

Assumption 1. Suppose $f_{e}(\cdot)$ is a measurable locally essentially bounded function satisfying the following property: There exist $\gamma_{f} \geq 0, \gamma_{e} \geq 0$ such that the contact force due to the environment, $f_{e}(t)$, satisfies the following two properties:

i) uniform boundedness: there exists $C \geq 0$ such that for any $t_{0} \in \mathbb{R}$

$$
\sup _{t \geq t_{0}}\left|f_{e}(t)\right| \leq \max \left\{\begin{array}{c}
C, \gamma_{e}\left(\sup _{t \geq t_{0}} \mid \begin{array}{c}
q_{s}(t) \\
\dot{q}_{s}(t)
\end{array}\right) \\
\gamma_{f}\left(\sup _{t \geq t_{0}}\left|f_{\text {ext }}(t)\right|\right)
\end{array}\right\}
$$

ii) convergence:

$$
\limsup _{t \rightarrow+\infty}\left|f_{e}(t)\right| \leq \max \left\{\begin{array}{c}
\gamma_{e}\left(\limsup _{t \rightarrow+\infty}\left|\begin{array}{l}
q_{s}(t) \\
\dot{q}_{s}(t)
\end{array}\right|\right), \\
\gamma_{f}\left(\limsup _{t \rightarrow+\infty}\left|f_{\text {ext }}(t)\right|\right)
\end{array}\right\} .
$$

Here $q_{s}(t), \dot{q}_{s}(t)$ are the state variables (position and velocity) of the slave manipulator, and $f_{\text {ext }}$ is an arbitrary measurement essentially bounded function that represents an equivalent of all external forces imposed on the environment.

\section{Communication Protocol, Communication Delay}

The information about master's position is sent through the communication channel to the remotely located slave with communication delay $\tau_{f}(\cdot)$, i.e., the signal

$$
\hat{q}_{m}(t)=q_{m}\left(t-\tau_{f}(t)\right),
$$

is available on the slave side. On the other hand, the contact force due to environment is transmitted back from the slave to the master over the communication channel with communication delay $\tau_{b}(\cdot)$, i.e., the following signal

$$
\hat{f}_{e}(t)=f_{e}\left(t-\tau_{b}(t)\right)
$$

is sent to the motors of the master. Both $\tau_{f}(\cdot), \tau_{b}(\cdot)$ are assumed to be time-varying and possibly unbounded. In this paper, we utilize two different assumptions on communication delay. First, we assume that the communication protocol in the forward communication channel guarantees sufficient smoothness of the communication delay function. More precisely, the following assumption is imposed on communication delay $\tau$.

Assumption 2. The communication delay $\tau: \mathbb{R} \rightarrow \mathbb{R}^{+}$, is a continuously differentiable function of time $t$ with the following properties:

i) $-\Upsilon_{1} \leq \frac{d \tau_{i}(t)}{d t} \leq 1$ for some $\Upsilon_{1} \geq 0$;

ii) $\left|\frac{d \tau_{i}}{d t}\left(t_{2}\right)-\frac{d \tau_{i}}{d t}\left(t_{1}\right)\right| \leq \Upsilon_{2}\left|t_{2}-t_{1}\right|$ for some $\Upsilon_{2} \geq 0$ and for all $t_{1}, t_{2} \in \mathbb{R}$;

iii) $t-\tau_{i}(t) \rightarrow+\infty$ as $t \rightarrow+\infty$. $\bullet$

On the other hand, we also address a situation where an unreliable communication protocol is utilized which admits discontinuity of the communication delay as a function of time. In this case, the following assumption is imposed.

Assumption 3. The communication delay $\tau_{i}: \mathbb{R} \rightarrow \mathbb{R}^{+}$, $i \in\{f, b\}$, is a piecewise continuous function with the following properties:

i) there exists $\Upsilon_{1} \geq 0$ such that the inequality

$$
-\Upsilon_{1}\left(t_{2}-t_{1}\right) \leq \tau_{i}\left(t_{2}\right)-\tau_{i}\left(t_{1}\right) \leq t_{2}-t_{1}
$$

holds for any $t_{1}, t_{2} \in \mathbb{R}^{n}, t_{2}>t_{1}$, such that $\tau_{i}(t)$ is continuous on $\left(t_{1}, t_{2}\right)$;

ii) $t-\tau_{i}(t) \rightarrow+\infty$ as $t \rightarrow+\infty$. $\bullet$

\section{Control Scheme}

In this section, let us present our control strategy for the force reflecting teleoperator system. The control scheme consists of local controllers on the master and the slave sides. The control algorithm for the master manipulator is as follows

$$
\begin{aligned}
u_{m}= & -H_{m}\left(q_{m}\right) \Lambda_{m} \dot{q}_{m}-C_{m}\left(q_{m}, \dot{q}_{m}\right) \Lambda_{m} q_{m} \\
& +G_{m}\left(q_{m}\right)-K_{m}\left(\dot{q}_{m}+\Lambda_{m} q_{m}\right),
\end{aligned}
$$


where $K_{m} \in \mathbb{R}^{n \times n}$ and $\Lambda_{m} \in \mathbb{R}^{n \times n}$ are symmetric positive definite matrices. On the other hand, the control algorithm for the slave manipulator is given below

$$
\begin{aligned}
\dot{\xi}_{1}= & \xi_{2}+g \alpha_{1}\left(\hat{q}_{m}-\xi_{1}\right) \\
\dot{\xi}_{2}= & g^{2} \alpha_{0}\left(\hat{q}_{m}-\xi_{1}\right) \\
u_{s}= & H_{s}\left(q_{s}\right)\left(g^{2} \alpha_{0}\left(\hat{q}_{m}-\xi_{1}\right)\right. \\
& \left.+\Lambda_{s}\left(\xi_{2}+g \alpha_{1}\left(\hat{q}_{m}-\xi_{1}\right)-\dot{q}_{s}\right)\right) \\
& +C_{s}\left(q_{s}, \dot{q}_{s}\right)\left(\xi_{2}+\Lambda\left(\xi_{1}-q_{s}\right)\right) \\
& +G_{s}\left(q_{s}\right)-K_{s}\left(\dot{q}_{s}-\xi_{2}+\Lambda\left(q_{s}-\xi_{1}\right)\right),
\end{aligned}
$$

where $K_{s} \in \mathbb{R}^{n \times n}, \Lambda_{s} \in \mathbb{R}^{n \times n}$ are symmetric positive definite matrices, $\alpha_{0}, \alpha_{1}$ are positive constants such that the roots of $p(s)=s^{2}+\alpha_{1} s+\alpha_{0}$ have negative real parts, and $g>0$ is a constant.

Remark 1. It is easy to see that the control algorithm for the slave manipulator includes a "dirty-derivative" filter (6), (7). The purpose of introducing the filter (6), (7) into the control algorithm is twofold. First, when the reference signal $\hat{q}_{m}$ is sufficiently smooth (which generally corresponds to the case of using a reliable communication protocol), the filter works as a (reduced-order) observer providing estimates for the time derivatives (first and second) of $\hat{q}_{m}$. On the other hand, when the reference signal is discontinuous (which may happen if the communication protocol admits data packets dropouts), the filter (6), (7) operates as a "governor" (see for example [12]) providing a smooth approximation of a possibly discontinuous reference trajectory $\hat{q}_{m}$.

\section{Main Results}

\section{A. Lossless Communication Protocol}

In this subsection, we study the stability and tracking properties of the controlled telerobotic system under the assumption that the communication protocol in the forward communication channel (from the master to the slave) guarantees sufficient smoothness of the communication delay function. More precisely, we assume that the function $\tau_{f}(\cdot)$ that represents the communication delay in the forward channel satisfies the assumption 2. For this case, it is convenient to choose the state of the telerobotic system as follows

$$
\mathbf{x}_{t}:=\left(q_{m}^{T}, \dot{q}_{m}^{T}, \tilde{\xi}_{1}^{T}, \tilde{\xi}_{2}^{T}, \tilde{q}_{s}^{T}, \tilde{\dot{q}}_{s}^{T}\right)_{t}^{T},
$$

where $\tilde{\xi}_{1}=\hat{q}_{m}-\xi_{1}, \tilde{\xi}_{2}=\dot{\hat{q}}_{m}-\xi_{2}, \tilde{q}_{s}:=q_{s}-\xi_{1}$, and $\tilde{\dot{q}}_{s}:=\dot{q}_{s}-\xi_{2}$, and where we use the notation $x_{t}(s):=x(t-$ $s), s \in\left[0, t_{d}(t)\right], t_{d}(t) \geq 0$. The input of the telerobotic system is

$$
u:=\left(f_{h}^{T}, f_{e x t}^{T}\right)^{T} .
$$

Also, for our purposes, it is convenient to define the following output of the controlled telerobotic system

$$
y:=\left(\tilde{\xi}_{1}^{T}, \tilde{\xi}_{2}^{T}, \tilde{q}_{s}^{T}, \tilde{\dot{q}}_{s}^{T}\right)^{T} .
$$

The main result of this section can be formulated as follows.

Theorem 1. Consider the force-reflecting telerobotic system (1), (2), (3), (4) under the controls (5), (6), (7), (8). Suppose the contact force due to environment $f_{e}$ satisfies Assumption 1. Further, suppose the communication delay $\tau_{f}(\cdot)$ in the forward channel (3) satisfies Assumption 2, while the communication delay $\tau_{b}(\cdot)$ in the backward channel (3) satisfies Assumption 3. Then the closed-loop teleoperator system is semiglobally inputto-state stable. More precisely, given positive constants $\gamma_{s}, \Delta_{x m}, \Delta_{x s}, \Delta_{f m}, \Delta_{f s}$, there exist $\kappa_{m} \geq 0, \kappa_{s} \geq 0$, and $g^{*}>0$ (all independent on $\left.\tau_{m}(\cdot), \tau_{s}(\cdot)\right)$ such that if $K_{m}$ in (5) satisfies $\lambda_{\min }\left(K_{m}\right) \geq \kappa_{m}, g$ in (6), (7) satisfies $g \geq g^{*}$, and $K_{s}$ in (8) satisfies $\lambda_{\min }\left(K_{m}\right) \geq \kappa_{m}$, then the closedloop telerobotic system with state (9) and input (10) is input-to-state stable at $t=0$ in the sense of definition 1 (see Appendix A) with

$$
t_{d}=\max \left\{\begin{array}{c}
\tau_{f}(0)+\tau_{b}\left(-\tau_{f}(0)\right), \\
\tau_{b}(0)+\tau_{f}\left(-\tau_{b}(0)\right)
\end{array}\right\},
$$

and restriction $\left(\Delta_{x m}, \Delta_{x s}, \Delta_{f m}, \Delta_{f s}\right)$. Moreover, the IOS gain with respect to output (11) is less than or equal to $\gamma_{s}$.

Remark 2. The results of Theorem 1 can be interpreted as follows. First, the stability of the overall system is guaranteed. Second, the IOS gain with respect to output (11) can be assigned arbitrarily small which means that the slave manipulator tracks the (delayed) master trajectory with an error bounded by an arbitrarily prescribed bound.

\section{B. Communication Protocol without Retransmission}

In this subsection, we study the properties of the proposed scheme in the situation where the communication is unreliable between the master and the slave manipulators. In fact, in some telerobotic applications, it may be desirable to always transmit the most recent available information about the master's position to the slave side. In this case, if some packets of information are lost during the transmission, they are usually not sent again. This may result in discontinuity of the communication delay function, which generally implies the discontinuity of the reference trajectory for the slave manipulator. Although in this case perfect tracking cannot be achieved because of such a possible discontinuity of the reference trajectory, however, as shown below, the proposed control scheme still guarantees the stability. To formulate the result, let us introduce a new set of state variables as follows

$$
\mathbf{x}_{t}:=\left(q_{m}^{T}, \dot{q}_{m}^{T}, \xi_{1}^{T}, \xi_{2}^{T}, \tilde{q}_{s}^{T}, \tilde{\dot{q}}_{s}^{T}\right)_{t}^{T} .
$$

The main result of this subsection is given in the following theorem.

Theorem 2. Consider the force-reflecting telerobotic system (1), (2), (3), (4) under the controls (5), (6), (7), (8). Suppose the contact force due to environment $f_{e}$ satisfies Assumption 1. Further, suppose the delays 
$\tau_{f}(\cdot), \tau_{b}(\cdot)$ in both the forward and the backward communication channels (3), (4) satisfy Assumption 3. Then the closed-loop teleoperator system with state (13) and input (10) is input-to-state stable at $t=0$ in the sense of definition 1 (see Appendix A) with $t_{d}$ defined by (12).

\section{Simulations}

In this section, we present some results of simulations. We have simulated a force-reflecting telerobotic system, where both the master and the slave are identical 2-DOF manipulators with $H_{m}(q)=H_{s}(q) \in \mathbb{R}^{2 \times 2}$, where

$$
\begin{aligned}
h_{11} & =\left(2 l_{1} \cos q_{2}+l_{2}\right) l_{2} m_{2}+l_{1}^{2}\left(m_{1}+m_{2}\right), \\
h_{12} & =h_{21}=l_{2}^{2} m_{2}+l_{1} l_{2} m_{2} \cos q_{2}, \\
h_{22} & =l_{2}^{2} m_{2}, \\
C_{m}(q, \dot{q})= & C_{s}(q, \dot{q}) \in \mathbb{R}^{2 \times 2}, \text { where } \\
c_{11} & =-l_{1} l_{2} m_{2} \sin \left(q_{2}\right) \dot{q}_{2}, \\
c_{12} & =-l_{1} l_{2} m_{2} \sin \left(q_{2}\right)\left(\dot{q}_{1}+\dot{q}_{2}\right), \\
c_{21} & =l_{1} l_{2} m_{2} \sin \left(q_{2}\right), \\
c_{22} & =0,
\end{aligned}
$$

and $G_{m}(q)=G_{s}(q) \in \mathbb{R}^{2}$, where

$$
\begin{aligned}
& g_{1}=g\left(m_{2} l_{2} \sin \left(q_{1}+q_{2}\right)+\left(m_{1}+m_{2}\right) l_{1} \sin \left(q_{1}\right)\right), \\
& g_{2}=g m_{2} l_{2} \sin \left(q_{1}+q_{2}\right),
\end{aligned}
$$

$m_{1}=10, m_{2}=5, l_{1}=0.7, l_{2}=0.5, g=9.81$. The forces (torques) applied by a human operator to the master manipulator are plotted in figure 1. When slave follows the resulting trajectiory of the master, it hits an obstacle which is located at $x=0.2 \mathrm{~m}$. The $\mathrm{x}$-component of the contact force due to contact with the obstacle is described by the following equations

$$
\begin{gathered}
F_{x}=0 \quad \text { if } x<0.2 m, \\
F_{x}=-B \dot{x}-K(x-0.2) \quad \text { if } x \geq 0.2 m,
\end{gathered}
$$

where $B \geq 0$ and $K \geq 0$ are damping and stiffness coefficients respectively. In the simulations below, we consider both the contacts with a soft $(K=100 \mathrm{~N} / \mathrm{m})$ and a rigid $(K=10000 N / m)$ environment respectively. In both the cases we put $B=1 \mathrm{~N} \cdot \mathrm{sec} / \mathrm{m}$. The parameters of the control law for the master manipulator (5) are set to be $K_{m}=5 \cdot \mathbb{I}^{2 \times 2}, \Lambda_{m}=5 \cdot \mathbb{I}^{2 \times 2}$, while, for the slave manipulator, the parameters of the control law (6)-(8), are as follows: $K_{s}=\operatorname{diag}\{10,5\}, \Lambda_{s}=\operatorname{diag}\{2,1\}, \alpha_{0}=1$, $\alpha_{1}=2$, and $g=10$.

In the first set of simulations, the communication delay function is choosen as a sum of constant signal $\tau_{1}=0.5 \mathrm{sec}$ and a white noise band-limited by a zeroorder hold with sampling period 0.1 sec. An example of such a communication delay function is plotted in figure 2. The corresponding simulation results for soft $(K=100 \mathrm{~N} / \mathrm{m})$ and rigid $(K=10000 \mathrm{~N} / \mathrm{m})$ environments are presented in figures 3,4 and 5, 6 respectively. In particular, $x$-components of the master and the slave trajectories as well as the $x$-component of contact force due to environment are shown in figure 3 for low stiffness and in figure 5 for high stiffness environment. In figures 4 and 6 the corresponding trajectories of the master and the slave in the joint space are shown as well as the corresponding $\xi_{1}$-trajectories of the dirty derivative filter (6), (7).

In the second set, simulations are performed in the presence of a communication delay function which is a sum of a discontinuous repeating sequence and a band-limited white noise, as shown in figure ??. Again, simulations are performed for both soft $(K=100 \mathrm{~N} / \mathrm{m})$ and rigid $(K=10000 N / m)$ environments. The corresponding results are shown in figures $7-10$. From the simulations presented, it is clear that in all the cases under consideration, the closed-loop teleoperator system demonstrates stable behaviour during contact with both soft and rigid environments. Moreover, the teleoperator system shows good tracking properties along pieces of trajectories that corresponds to motion in free space.

\section{Concluding remarks}

In this paper, the problem of force-reflecting teleoperation over IP networks is addressed. Taking into account time-varying communication delay and possible data packets dropout, we propose a control scheme that guarantees the stability of the overall system. In the case where the communication delay in the forward communication channel is guaranteed to be smooth enough function of time, the proposed scheme also guarantees that the slave tracks the delayed master trajectory with an error bounded by a prescribed constant. A possible extension of the proposed scheme may incorporate some sort of a force control algorithm on the slave side which may improve the performance of teleoperation. On the other hand, the master control algorithm can also be modified to incorporate an estimate of the remote environment dynamics, as in [15]. These will be topics for future research.

\section{References}

[1] W. R. Ferrell, "Delayed force feedback," Human Factors, vol. 8, pp. 449-455, 1966

[2] R. J. Anderson and M. W. Spong, "Bilateral control of teleoperators with time delay," IEEE Trans. Aut.Contr., vol. AC-34, pp. 494-501, May 1989.

[3] G. Niemeyer and J.-J. E. Slotine, "Stable adaptive teleoperation," IEEE Journal of Oceanic Engineering, vol. 16, pp. 152162, Jan. 1991

[4] G. M. Leung, B. A. Francis, and J. Apkarian, "Bilateral controller for teleoperators with time delay via mu-synthesis," IEEE Trans. Robotics and Automation, vol. 11, pp. 105-116, 1995.

[5] P. Arcara and C. Melchiorri, "Control schemes for teleoperation with time delay: a comparative study," Robotics and Autonomous Systems, vol. 38, pp. 49-64, Jan. 2002.

[6] G. Niemeyer and J.-J. E. Slotine, "Towards force reflecting teleoperation over the internet," in International Conference on Robotics and Automation, (Leuven, Belgium), pp. 19091915,1998 
[7] N. Chopra, M. W. Spong, S. Hirche, and M. Buss, "Bilateral teleoperation over the internet: the time varying delay problem," in American Control Conference, (Denver, CO), June 2003.

[8] W. Zhang, M. S. Branicky, and S. M. Phillips, "Stability of networked control systems," IEEE Control Systems Magazine, vol. 21, pp. 84-99, Feb. 2001.

[9] I. G. Polushin, A. Tayebi, and H. J. Marquez, "Stabilization scheme for force reflecting teleoperation with time-varying communication delay based on IOS small gain theorem," in 16th IFAC World Congress, (Prague, Czech Republic), 2005. In press.

[10] M. W. Spong, "Motion control of robot manipulators," in Handbook of Control (W. Levine, ed.), pp. 1339-1350, CRC Press, 1996.

[11] E. D. Sontag and Y. Wang, "Notions of input-to-output stability," Systems \& Control Letters, vol. 38, pp. 235-248, 1999.

[12] E. Gilbert and I. Kolmanovsky, "Nonlinear tracking control in the presence of state and control constraints: A generalized reference governor," Automatica, vol. 38, pp. 2063-2073, 2002.

[13] E. D. Sontag and Y. Wang, "On characterizations of the inputto-state stability property," Systems \& Control Letters, vol. 24, pp. 351-359, 1995.

[14] G. E. Shilov, Elementary Functional Analysis. Cambridge, Mass.: MIT Press, 1974. translated and edited by Richard A. Silverman.

[15] L. J. Love and W. J. Book, "Force reflecting teleoperation with adaptive impedance control," IEEE Transactions on Systems, Man, and Cybernetics - Part B:Cybernetics, vol. 34, pp. 159166, Feb. 2004.

[16] J. K. Hale, Theory of Functional Differential Equations. New York: Springer-Verlag, 1977.

[17] A. R. Teel, "Connections between Razumikhin-type theorems and the ISS nonlinear small gain theorem," IEEE Transactions on Automatic Control, vol. AC-43, pp. 960-964, July 1998.

Appendix A. Input-to-output stability for FDE

Given a function $x: \mathbb{R} \rightarrow \mathbb{R}^{n}$, denote $x_{t}(s):=x(t-s)$, where $s \geq 0$. Consider a system of functional differential equations with $l$ inputs and $r$ outputs of the following form

$$
\begin{aligned}
\dot{x}(t) & =F\left(x_{t}, u_{t}^{\{1\}}, \ldots, u_{t}^{\{l\}}, d_{t}\right), \\
y^{\{1\}}(t) & =H^{\{1\}}\left(x_{t}, u_{t}^{\{1\}}, \ldots, u_{t}^{\{l\}}, d_{t}\right), \\
\cdots & \\
y^{\{r\}}(t) & =H^{\{r\}}\left(x_{t}, u_{t}^{\{1\}}, \ldots, u_{t}^{\{l\}}, d_{t}\right),
\end{aligned}
$$

Here $x$ is the state, $u^{\{i\}}, i \in\{1, \ldots, l\}$ are the inputs, $y^{\{j\}}, j \in\{1, \ldots, r\}$ are the outputs, and $d(\cdot)$ are the perturbations that are elements of the set of admissible perturbations $\mathcal{D}$. It is assumed that both $F$ and $H$ are continuous operators in $x_{t}, u_{t}$, and $d_{t}$. In particular, this guarantees the existence and uniqueness of solutions as well as continuous dependence of the solutions in $x_{t}$, $u_{t}[16]$.

The following definition presents a version of the notion of input-to-output stability specified for multiinput multi-output systems of FDE. A close definition of input-to-state stability (ISS) for FDE was introduced in [17].

Definition 1. System of the form (14) is said to be inputto-output stable (IOS) at the moment $t=0$ with $t_{d} \geq 0$, IOS gains $\gamma^{\{i j\}} \in \mathcal{K}, i \in\{1, \ldots, l\}, j \in\{1, \ldots, r\}$, and restriction $\left(\Delta_{x}, \Delta_{u}^{\{1\}}, \ldots, \Delta_{u}^{\{l\}}\right) \in \mathbb{R}_{>0}^{l+1}$, if the conditions $\sup _{t \in\left[-t_{d}, 0\right]}|x(t)| \leq \Delta_{x}$, and $\sup _{t>-t_{d}}\left|u^{\{i\}}(t)\right| \leq \Delta_{u}^{\{i\}}, i \in$ $\{1, \ldots, l\}$ imply that the solutions of (14) are well-defined for all $t \in[0,+\infty)$, and the following two properties hold:

i) uniform boundedness: there exists a function $\beta \in \mathcal{K}_{\infty}$ and $C \geq 0$ such that

$$
\sup _{t \geq 0}\left|y^{\{j\}}(t)\right| \leq \max \left\{\begin{array}{c}
\beta\left(\sup _{s \in\left[-t_{d}, 0\right]}|x(s)|\right), \\
\gamma^{\{1 j\}}\left(\sup _{s \geq-t_{d}}\left|u^{\{1\}}(s)\right|\right), \\
\ldots, \\
\gamma^{\{l j\}}\left(\sup _{s \geq-t_{d}}\left|u^{\{l\}}(s)\right|\right), C
\end{array}\right\}
$$

for all $j \in\{1, \ldots, r\}$;

ii) convergence:

$\limsup _{t \rightarrow \infty}\left|y^{\{j\}}(t)\right| \leq \max \left\{\begin{array}{c}\gamma^{\{1 j\}}\left(\limsup _{t \rightarrow \infty}\left|u^{\{1\}}(t)\right|\right), \\ \ldots, \\ \gamma^{\{l j\}}\left(\limsup _{t \rightarrow \infty}\left|u^{\{l\}}(t)\right|\right)\end{array}\right\}$

for all $j \in\{1, \ldots, r\}$.

In this case, a function $\gamma^{\{i, j\}} \in \mathcal{K}$, where $i \in\{1, \ldots, l\}$, $j \in\{1, \ldots, r\}$, is called the IOS gain from $u^{\{i\}}$ to $y^{\{j\}}$.

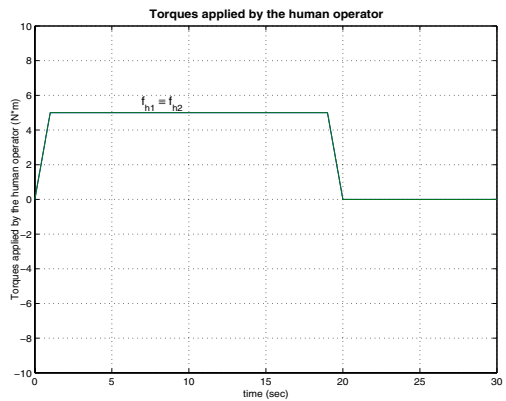

Fig. 1. Forces (torques) applied by the human operator
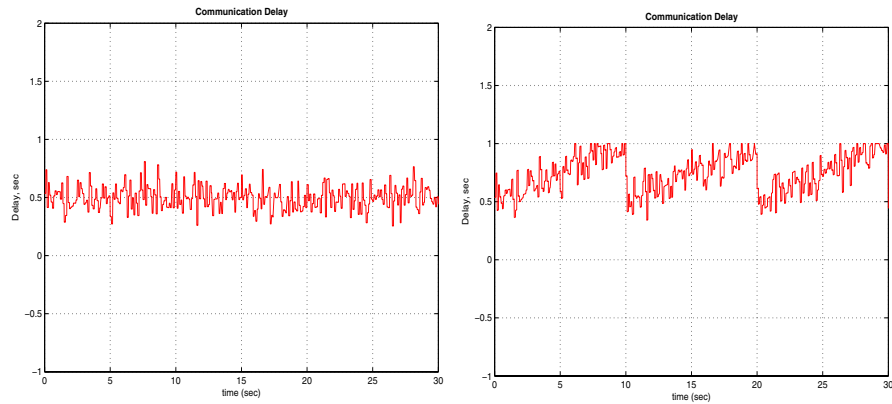

Fig. 2. Communication Delay Functions, from left to right: i) 1st set of simulations; ii) 2nd set of simulations 

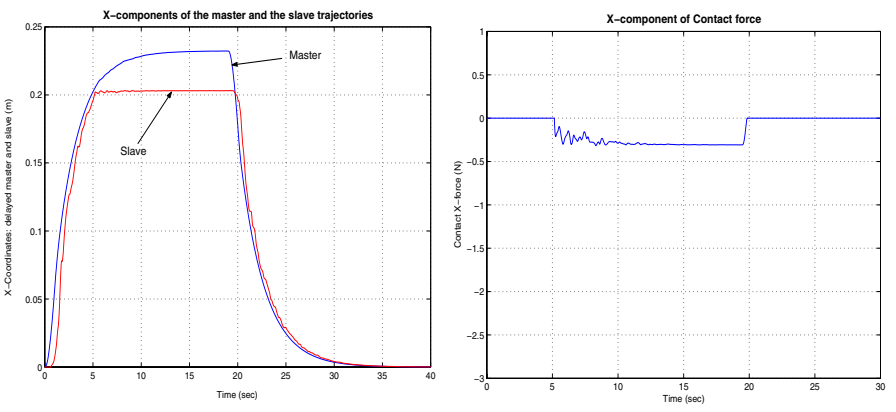

Fig. 3. 1st set of simulations, low stiffness $100 \mathrm{~N} / \mathrm{m}$, from left to right: i) X-trajectories of the master and the slave; ii) X-component of contact forces
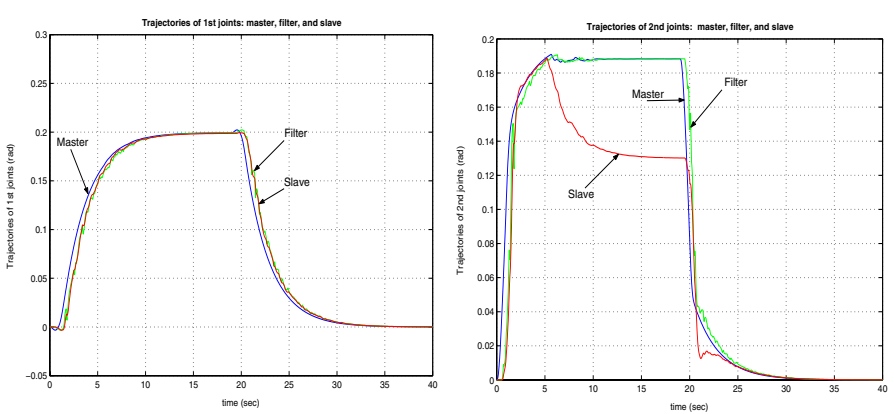

Fig. 4. 1st set of simulations, low stiffness $100 \mathrm{~N} / \mathrm{m}$. Trajectories in the joint space, from left to right: i) 1 st joints; ii) 2 nd joints
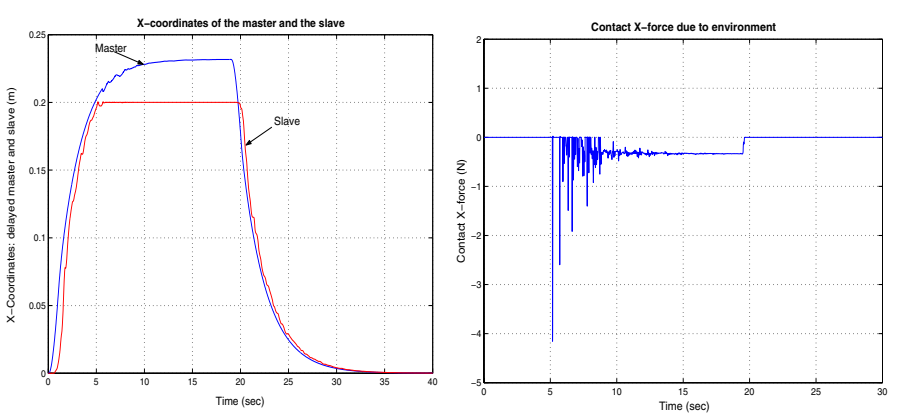

Fig. 5. 1st set of simulations, high stiffness $10000 \mathrm{~N} / \mathrm{m}$, from left to right: i) X-trajectories of the master and the slave; ii) X-component of contact forces
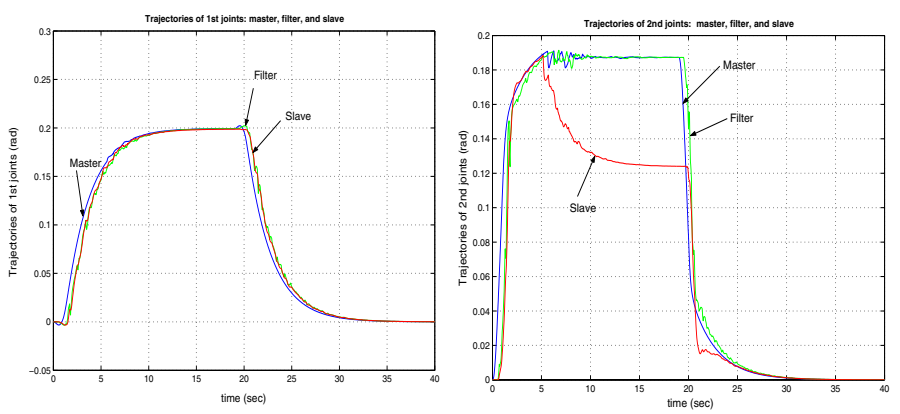

Fig. 6. 1st set of simulations, high stiffness $10000 \mathrm{~N} / \mathrm{m}$. Trajectories in the joint space, from left to right: i) 1st joints; ii) 2 nd joints
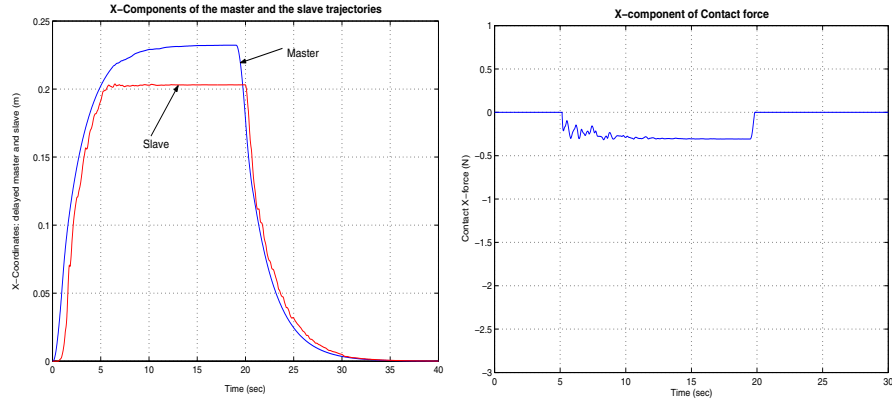

Fig. 7. 2nd set of simulations, low stiffness $100 \mathrm{~N} / \mathrm{m}$, from left to right: i) X-trajectories of the master and the slave; ii) X-component of contact forces
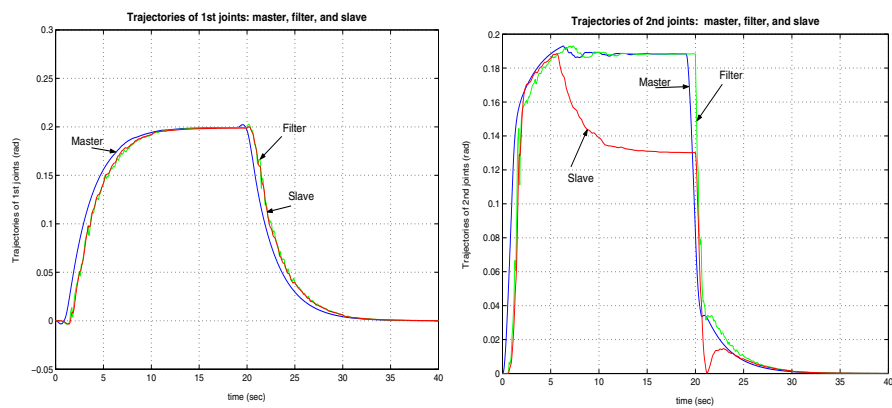

Fig. 8. 2nd set of simulations, low stiffness $100 \mathrm{~N} / \mathrm{m}$. Trajectories in the joint space, from left to right: i) 1st joints; ii) 2nd joints
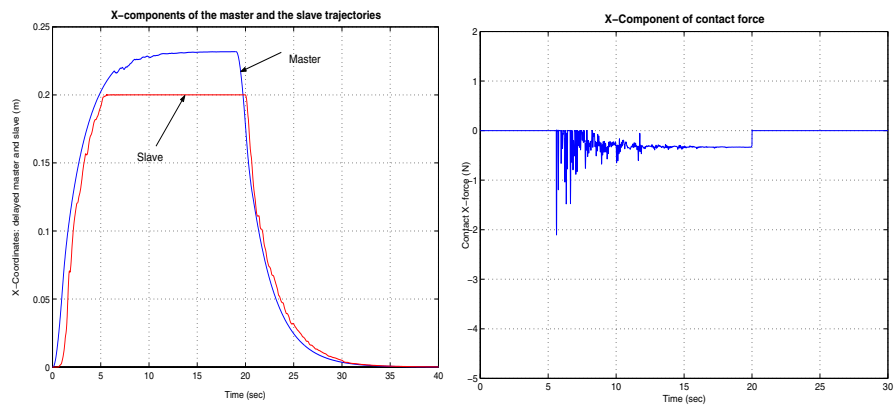

Fig. 9. 2nd set of simulations, high stiffness $10000 \mathrm{~N} / \mathrm{m}$, from left to right: i) X-trajectories of the master and the slave; ii) X-component of contact forces
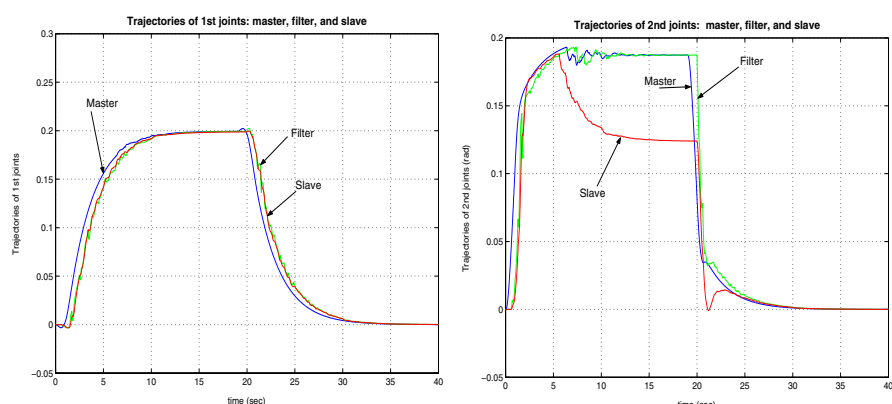

Fig. 10. 2nd set of simulations, high stiffness $10000 \mathrm{~N} / \mathrm{m}$. Trajectories in the joint space, from left to right: i) 1st joints; ii) 2nd joints 